\title{
Epistemología crítica para qué: los desafíos de la educación en el contexto de la crisis global del capitalismo
}

Daniel Carlos Gutiérrez Rohán*

\begin{abstract}
Resumen. En el contexto de la crisis terminal del modelo neoliberal, que ha generado pobreza y desigualdad extremas en todo el mundo, en donde debido a la injustica social, la corrupción, la impunidad en todos los niveles, la inoperancia de las estructuras y actores políticos, es común la desesperanza. Se ha dicho de manera insistente que la educación debe convertirse en el instrumento privilegiado para superar la crisis y promover el desarrollo de las sociedades mundiales de una manera mucho más equilibrada; sin embargo, en nuestro país esto aún se encuentra como mero discurso, debido al gran peso de las estructuras político-administrativas y su capacidad de control e imposición, pero principalmente, a la ausencia de motivaciones críticas de quienes tienen la responsabilidad de hacer operativos los procesos educativos: los maestros. Y esto vale para cualquier nivel educativo, desde la educación básica hasta los posgrados.
\end{abstract}

Doctor en Ciencias Políticas de la Universidad Nacional Autónoma de México. Investigador y coordinador nodo México de la Red Latinoamericana de Metodologías de las Ciencias Sociales dcgutier@sociales.uson.mx 
Palabras clave. Epistemología crítica, globalización, capitalismo, educación.

\begin{abstract}
In the context of the terminal crisis of the neoliberal model that has generated extreme poverty and inequality around the world; where, due to social injustice, corruption, impunity at all levels, the failure of structures and political actors, hopelessness is common. It has been said insistently that education must become the instrument for overcoming the crisis and promote global development of societies in a much more balanced way, but in our country it has not became reality yet, due to great weight of the political and administrative structures and their ability to control and to enforce, but mainly due to the lack of critical motivation from the ones who have the responsibility of operating the educational process: the teachers. And this applies to all educational levels, from basic education to postgraduate studies.
\end{abstract}

Keywords. Critical epistemology, globalization, capitalism, education.

\title{
Introducción
}

Dice Gastón Bachelard que

un conocimiento adquirido por un esfuerzo científico puede declinar. La pregunta abstracta y franca se desgasta, la pregunta concreta queda. Con esto la actividad espiritual se invierte y se endurece... la idea conquista así una claridad intrínseca abusiva. Con el uso las ideas se valorizan indebidamente... un epistemólogo irreverente declara que los grandes hombres son útiles a la ciencia (y a la docencia diríamos nosotros en este caso) en la primera mitad de su vida nocivos en la segunda... llega el momento en que el espíritu prefiere lo que confirma su saber a lo que lo contradice, en el que prefiere las respuestas 
a las preguntas. Entonces el espíritu conservativo domina, y el crecimiento espiritual se detiene (1999, p.17).

Esta referencia es importante para repensar nuestra propia práctica académica, sea en el ámbito de la investigación o sea en el de la docencia, o en ambos, tal y como se plantean las funciones de los profesores en la mayoría de las universidades públicas en México.

El asunto aquí es preguntarnos iqué tanto de nuestra propia práctica docente se refleja en lo que plantea Bachelard? ¿En qué proporción de nuestro quehacer preferimos la respuesta sobre la pregunta? ¿Hasta dónde consideramos que nuestros saberes son suficientes para dar respuesta a cualquier pregunta?

La realidad es que nuestra propia práctica académica se encuentra permeada por un conjunto de determinaciones que nos han llevado a reproducir esquemas sin ningún tipo de cuestionamiento; incluso hemos transformado el lenguaje académico para expresarnos a través de acrónimos, de fórmulas lingüísticas y conceptos derivados de los programas de gobierno. Así, ya no son facultades, escuelas o departamentos sino DES (Dependencia de Educación Superior), anualmente hay que elaborar los PIFIs (Programa Integral de Fortalecimiento Institucional), obtener el perfil PROMEP (Programa de Mejoramiento del profesorado), renovar o ingresar al SIN (Sistema Nacional de Investigadores), etcétera; o bien el modelo por competencias, mejoramiento de la calidad académica, indicadores de retención, eficiencia terminal, entre otros. Esta nueva estructura lingüística se ha ido asimilando hasta convertirse en una forma cotidiana de intercambio de información, sin ningún tipo de análisis o reflexión en función de las propias disciplinas, porque lo que se discute no son los contenidos disciplinares de tal o cual campo de conocimiento, sino que se asumen desde una lógica administrativa. Lo cual ha significado someter los procesos académicos a las necesidades administrativas de los cuerpos directivos de las universidades.

ITINERARIO EDUCATIVO • AÑO XXVI, N. ${ }^{\circ} 59 \cdot 3 I-5 I \cdot$ • ENERO - JUNIO DE ZOIS 
Hay profesores, por lo menos en la universidad donde yo trabajo, que se muestran satisfechos con estas orientaciones, de tal suerte que delimitan sus esfuerzos académicos a responder de manera puntual a los requerimientos impuestos por los programas federales (hablamos del Sistema Nacional de Investigadores, del PROMEP, de los programas de estímulos, etcétera), y esto es entendible pues están asociados a compensaciones económicas. Pero también están asociados a las visiones de lo que la investigación o la docencia deben ser. Desde luego en esas visiones no caben los cuestionamientos ni sobre el papel del profesor ni sobre las características o fundamentos de esos programas, pues de lo que se trata es, justamente, hacer prevalecer esas orientaciones. Finalmente, si los profesores no quieren o no pueden participar en esos programas, no pasa nada, simplemente sus ingresos no aumentan. Así que hay que olvidarse de la crítica y del cuestionamiento y asumir tal cual las condiciones institucionales.

Esto es el inicio de una posible explicación de por qué lo que impera en nuestro medio académico es el predominio de la respuesta sobre la pregunta, en donde «el espíritu conservativo domina, y el crecimiento espiritual se detiene».

Sin embargo, aunque esto pueda constituir una explicación, no representa una causa sino que es el resultado de, por lo menos, las siguientes cuestiones históricas:

1. Un modelo económico depredador; un capitalismo salvaje cuyo esquema de acumulación ha sometido no sólo los procesos económicos sino también los políticos, sociales y, en este marco, los procesos educativos a los intereses de la oligarquía mundial. Un modelo que actualmente se encuentra sumido en una crisis global.

2. El predominio de una visión occidentalizadora sobre los procesos económicos, políticos, sociales y educativos, sin considerar las condiciones y necesidades de cada país. 
3. La cultura occidental como único referente en el quehacer académico, tanto en investigación como en la docencia, que tiene sus contenidos teóricos, metodológicos, interpretativos y los modelos de enseñanza en los centros académicos y científicos de los países hegemónicos.

Como puede observarse, de acuerdo a lo anterior, en México también vivimos una crisis social como parte de la crisis que se vive a escala global y que afecta al conjunto de esferas de la vida social. Esto constituye el fondo para entender que la problemática por la que atraviesa la educación no tiene como causa los bajos niveles académicos o los indicadores de calidad impuestos por organismos internacionales, sino que se enmarcan como parte de un proceso histórico que ha definido desde nuestros puntos de observación hasta nuestras propias prácticas académicas; que ha detenido el crecimiento del espíritu científico, del espíritu crítico al que se aludió en la cita de Bachelard.

\section{§ I}

En lo que respecta a la crisis del modelo neoliberal ya algunos autores importantes (Wallerstein, Bauman, De Sousa, Emir Sader, Zemelman, entre otros) y las propias autoridades del Banco Mundial (BM), del Fondo Monetario Internacional (FMI) o la propia Organización para la Cooperación y el Desarrollo Económico (OCDE), en su momento, han expresado que la etapa neoliberal del desarrollo del capitalismo es un modelo en fase terminal.

De este proceso de crisis hay dos fenómenos que llaman poderosamente la atención. En primer lugar, la resistencia de las oligarquías mundiales a renunciar a las formas de organización de la economía que acrecentaron enormemente su riqueza y como contraparte se acrecentó la pobreza de la mayoría de la población mundial. Son estas minorías quienes están generando reacciones que buscan impedir la cristalización de nuevas opciones para

ITINERARIO EDUCATIVO • AÑO XXVI, N. ${ }^{\circ} 59 \cdot 3 I-5 I \cdot$ ENERO - JUNIO DE $20 I 2$ 
reorganizar, de manera equilibrada, el desarrollo de las sociedades nacionales.

Estas oligarquías son tan poderosas que han permeado con su influencia las esferas políticas de los países hegemónicos y, desde luego, a los gobiernos de países con poco desarrollo. Esta aparece como una razón de por qué todavía no se muestran de manera orgánica condiciones para un cambio. Es desde la esfera política desde donde tiene que iniciar la construcción de alternativas para el desarrollo social. En este contexto se entiende, en mucho, lo que ha estado sucediendo en algunos países del mundo árabe, como en Egipto, Siria, Libia, entre otros y lo que es manifestado en Europa, específicamente en Grecia y más recientemente en España. En México con la violencia de Estado y las aún inorgánicas respuestas sociales.

Un rasgo común de estas manifestaciones sociales es que no han cuestionado la estructura económica sino, justamente, quizá por la sensibilidad social, han dirigido los movimientos contra los gobiernos como la organización política de la sociedad. En este mismo sentido, no se puede dejar de considerar que en estos procesos se insertan distintos intereses, dentro de los que se incluyen, de manera prioritaria, los de las oligarquías, quienes a través de su capacidad de influir y corromper buscan impulsar, con el gobierno de Estados Unidos a la cabeza, un gatopardismo: cambiar para que nada cambie. En este contexto se articulan diversos momentos históricos; es decir, diferentes procesos con temporalidades distintas, que se encuentran aún en potencia, que aún no se expresan con suficiente madurez para dar lugar a lo posible, a nuevas condiciones de organización social, tal y como lo menciona Ernest Bloch (2007, p. 158):

Para que este novum pueda salir de la mera incubación y hacerse repentinamente lúcido tienen, pues, que estar puestas las condiciones, no solo subjetivas, sino también objetivas para la 
expresión de un novum y esas condiciones son siempre progresivas, de índole progresiva económica social.

Bloch también señala, y lo recuperamos para nuestro momento histórico, que «en estos tiempos el hombre se siente claramente como un ser no fijado definitivamente, como un ser que, junto a su entorno, constituye un cometido y un gigantesco recipiente lleno de futuro» (p.154).

De tal modo que, en este sentido, el aún no o el todavía no, tiene que ver con un movimiento molecular que no puede dar lugar a lo nuevo sino hasta que existan las condiciones para la ruptura de la continuidad, en este caso neoliberal. El gran problema es que aún no existe un modelo alternativo de mundo. Lo que ya existe es una cantidad enorme de procesos y fenómenos emergentes que sólo desde la esfera política (que no se refiere sólo al gobierno, sino, de manera amplia, a los espacios públicos) se pueden articular para orientarlos al futuro.

En segundo lugar, esta crisis de la etapa neoliberal del capitalismo, ha dado lugar, como se dijo arriba, a una gran cantidad de fenómenos novedosos, emergentes que aún no entendemos, que todavía no se pueden explicar; principalmente porque las formas de mirarlas corresponden a viejos paradigmas o bien a planteamientos inapropiados que ven más al pasado que al futuro.

Para estos procesos y fenómenos emergentes, en muchos casos, no se han generado las disposiciones para construir sujetos que estén en condiciones de formular nuevas preguntas, nuevos abordajes metodológicos para explicarlos. Bien sea porque se siguen empleando teorías, categorías, conceptos y metodologías que fueron útiles para otros momentos históricos, o porque aún no se han desarrollado todas las condiciones cuantitativas para dar un salto cualitativo. 
El hecho es que, en esta etapa de crisis, estamos atrapados en la ruptura, es decir, atrapados entre las resistencias de los grupos oligárquicos mundiales y nacionales para mantener sus márgenes de ganancia y sus posiciones privilegiadas y la influencia sobre los gobiernos subordinados a sus intereses y ante la dificultad de estructurar alternativas y horizontes nuevos pues, básicamente, aún no sabemos con certeza qué sigue después de esta etapa.

De una u otra forma, esta ausencia de alternativas reales para el mundo revela que, en efecto, la temporalidad de los procesos de ruptura en curso y que aún no han desarrollado todas las condiciones para dar un salto hacia nuevos procesos e imprimir un nuevo impulso al movimiento de la historia.

En los casos señalados, tanto en lo que se refiere a la resistencia de los grupos oligárquicos mundiales y nacionales, con toda su capacidad económica y de influencia política, como en la ausencia de opciones para superar la crisis global que vivimos, al parecer no estamos formulando las preguntas pertinentes para entender y explicar lo que se encuentra en potencia, lo todavía no, lo aún no blochiano, y los procesos y fenómenos emergentes que este movimiento de la historia está generando, porque la tendencia dominante es no cuestionar los puntos de observación ni las estructuras teóricas a las que se recurre en busca de explicaciones, es decir, a no modificar nuestro pensamiento y transformar las fórmulas que aprendemos y que, por comodidad intelectual o por legitimación profesional, mantenemos y aplicamos cotidianamente. Vemos solamente lo que nos enseñan a ver cuando lo que no vemos es incalculable y de una mayor riqueza, como lo dice De Sousa (2009).

Nos movemos en función de viejos paradigmas o nuevos, pero que no explican un mundo que se mueve y modifica de forma permanente (el mundo de la producción, de la política, de la cultura, de la educación); ya no podemos verlo desde esas lógicas 
paradigmáticas, sin por lo menos revisarlas críticamente. Transformar nuestras formas de generar conocimiento requiere de la construcción de nuevos sujetos y con ello transformar la relación sujeto-objeto, tanto en el ámbito de la investigación como en el de la docencia.

\section{$\S 2$}

Este movimiento de la historia en el mundo, se expresa de manera nítida en México y posiblemente en otros países de América Latina, tanto en lo que se refiere a la resistencia de los grupos oligárquicos como a los paradigmas desde donde se piensan todos los procesos. Los rasgos que se observan claramente son: 1) Un grupo reducido que concentra un enorme porcentaje de la riqueza nacional; 2) Un porcentaje inmenso de la población en condición de pobreza y de pobreza extrema; 3) Un desempleo creciente, principalmente para los jóvenes; 4) Un gobierno inoperante empeñado en una guerra sin sentido, con estructuras corrompidas y altos grados de impunidad; 5) Un gobierno subordinado incondicionalmente a los intereses norteamericanos; 6) Una crisis de legitimidad; 7) Estructuras institucionales permeadas por orientaciones provenientes de organismos internacionales (eufemismo de países hegemónicos), que se han traducido en políticas de gobierno; 8) Una crisis profunda de la educación en todos sus niveles.

Estos son algunos de los principales rasgos de la crisis del modelo neoliberal mexicano. Es en este marco de complejidad donde debemos enmarcar los procesos educativos en general, y de manera particular los relacionados con la educación superior para entender la dimensión de la crisis educativa y saber de qué hablamos cuando nos referimos a la problemática de la educación en cualquier estado de la República mexicana.

Queda claro que no es únicamente una cuestión de financiamiento (que si lo es y de manera relevante), sino también de las 
concepciones incorporadas en los programas que las instituciones de educación plantean como orientadoras de los procesos educativos. Son concepciones que responden a los lineamientos de la Organización para la Cooperación y el Desarrollo Económico (OCDE), de la cual México forma parte y por ello asume el compromiso de integrar sus lineamientos y recomendaciones. Por ejemplo las pruebas ENLACE (Evaluación Nacional del Logro Académico en Centros Escolares) y PISA (por sus siglas en inglés Program for International Student Assessment, Programa Internacional para la Evaluación de Estudiantes) y el gran impulso que las autoridades de la Secretaría de Educación Pública le han dado a la formación por competencias.

Bajo esta lógica, la educación de los mexicanos obedece a necesidades e intereses de instancias externas más que a necesidades propias de nuestro país, a las características y circunstancias regionales y locales. Esta situación es un componente importante de la crisis que junto con la corrupción y el corporativismo magisterial constituyen un lastre que no permite superar la crisis ya de tantos años y propiciar el desarrollo educativo de los mexicanos.

Además de esto, hay otro elemento fundamental que habría que considerar en este escenario: los cuerpos directivos y las plantas académicas, quienes han asumido estos lineamientos sin la menor reflexión y análisis. Sumados a esta lógica, hemos renunciado a ver a la educación, a los campos de conocimiento desde puntos de observación diferentes a los impuestos en las reformas impulsadas por las instancias oficiales (SEP -Secretaría de Educación Pública-, ANUIES -Asociación Nacional de Universidades e Instituciones de Educación Superior-, CONACYT -Consejo Nacional de Ciencia y Tecnología-). Hemos renunciado a plantearnos preguntas sobre la pertinencia de los programas, sobre los contenidos y saberes que enseñamos. Hemos asumido quedarnos con las respuestas que nos han dado. 
Esto constituye un problema central, pues al quedar excluida toda forma diferente a la visión oficial, nos instalamos en la inmovilidad, en la incapacidad para resolver los problemas de la educación y de nuestra propia práctica docente. De tal forma que, en muchos casos, hemos caído en la repetición de fórmulas y en la subordinación de la producción de conocimiento y los procesos de enseñanza aprendizaje a los lineamientos establecidos por las autoridades educativas federales, que pueden tener contenidos muy pertinentes, pero eso no lo reflexionamos.

Para ilustrar lo anterior, tomemos la propuesta de formación basada en competencias (que es un modelo que atraviesa prácticamente a todo el sistema educativo). Se ha propuesto una reforma al sistema Bachillerato, conocida como la RIEMS (Reforma Integral para la Educación Media Superior), que busca uniformar en todo el país los contenidos de planes y programas de estudio del bachillerato; la principal característica es que está basada en el modelo por competencias. Sin embargo, no queda claro de qué se habla cuando se habla de competencias. De todas las posibles aproximaciones (que son algunas) a la idea de competencia, prevalece aquella asociada a las visiones gerenciales, de estandarización, propias del mundo de la producción que buscan formar individuos eficientes en determinadas competencias. Esto quizá no esté mal, sobre todo para ciertas áreas de ingeniería, pero el problema es que ni siquiera lo reflexionamos de acuerdo a las exigencias de cada campo de conocimiento, ni nos preguntamos si este modelo es apropiado para nuestras condiciones y necesidades, si es pertinente con nuestra formación intelectual y profesional, ni mucho menos si es pertinente de acuerdo a las características y necesidades de cada región donde se ubican las instituciones, simplemente se asumió que así tenía que ser. ¿Qué significa esto? Más allá de lo que representa la subordinación directa de la educación a las necesidades de los procesos económicos, a través de la formación de una fuerza laboral dócil, significa que, en estas condiciones, 
estamos atrapados en la imposibilidad de dar un nuevo impulso y una nueva direccionalidad a la educación en nuestro país. Significa renunciar a pensar creativamente, críticamente en el sentido de exigir al pensamiento mayor rigor y profundidad. Significa renunciar a la búsqueda de innovaciones y alternativas educativas para con ello contribuir al desarrollo social, incluido el desarrollo económico. Significa postergar la búsqueda de alternativas para superar la crisis de la educación en México. Si la gente, en este caso los profesores, los investigadores, cuya función central es pensar, generar y transmitir conocimiento, no lo hacemos, entonces íquién lo hará?

Por otra parte, este modelo por competencias supone también una concepción en donde se prioriza el aprendizaje pero limitado por las competencias definidas para la formación académica; las cuales, de manera genérica se identifican, según Levin y Mourange (2004), las siguientes para una vida «exitosa y responsable»:

1. Las habilidades básicas de lectura y matemáticas son importantes para determinar los resultados laborales a largo plazo, incluyendo la capacidad para ajustarse a los cambios y circunstancias.

2. La capacidad de comunicarse efectivamente, tanto de manera oral como escrita, es importante para determinar los resultados laborales a largo plazo.

3. La organización del trabajo dentro de las empresas está cambiando de maneras tales que imprime cada vez un valor mayor a la capacidad de trabajar de manera productiva en grupos.

4. Los cambios en las empresas (con énfasis en el trabajo en equipo) y los cambios en la economía enfatizan cada vez más los elementos de la inteligencia emocional, incluyendo la capacidad de relacionarse con las demás personas. 
5. La familiaridad con las computadoras tiene una importancia creciente en el mercado laboral.

6. Los papeles que acreditan la educación formal tienen cada vez mayor importancia para determinar el éxito económico.

7. Las fuerzas del mercado que valoran las competencias clave se ven modificadas por diversos acuerdos institucionales: la organización del trabajo (...), los vínculos entre las empresas y las instituciones educativas, la medida en la que se regulan los mercados de una nación y la distribución nacional de los ingresos lo regula el Estado (p. 287-291).

Sobre bases de este tipo se han ido trasformando los planes y programas de estudio en un gran número de instituciones de educación superior, desde luego estimuladas y presionadas por las autoridades educativas del gobierno federal. De pronto, la educación se convierte en un proceso basado en competencias, sin que haya habido una reflexión seria y un análisis sobre lo que esto implica; todo lo contrario, se asumió como una cuestión innovadora por una buena parte del profesorado, sin que se comprendiera bien a bien de que se trataba este modelo para las propias plantas académicas formadas en métodos, estrategias didácticas y pedagógicas, digamos tradicionales, con los imperativos de incorporar las tecnologías de la información y la comunicación. De tal manera que se han generado en la práctica docente una suerte de mezcla entre una deficiente integración del modelo por competencias y una variedad amplia de estrategias didáctico-pedagógicas producto de las trayectorias académicas de los profesores.

El posgrado tampoco ha quedado al margen de estos procesos y como ejemplo basta referir las maestrías profesionalizantes, cuya pretensión es ligarlas directamente, como una forma de especialización, también a los procesos económicos. Esta problemática tiene 
un conjunto de implicaciones sobre lo que aún no (nuevamente en el sentido blochiano) hemos reflexionado lo suficiente.

Es necesario destacar, en primer lugar, que esa formación tradicional del profesorado es producto de un proceso de adquisición de saberes que de antemano determinan los puntos de observación y lo que hay que ver. Es decir, aprendemos a mirar desde la perspectiva de los paradigmas dominantes, o sea, desde la cultura occidental. Nos involucramos en procesos de enseñanza aprendizaje en los cuales ya sabemos de antemano lo que tenemos que ver y entonces lo que enseñamos y aprendemos es la manera de mirarlo y lo vemos como si lo estuviéramos viendo desde los países hegemónicos, pero en la condición de país con un pobre desarrollo social. Como lo alude De Sousa (2009), al sur lo vemos como si estuviéramos en el norte; eso es lo que aprendemos y eso es lo que enseñamos.

Entonces, enseñamos un conjunto de saberes cristalizados, una manera de ver la realidad que no cuestiona, fundada en certezas, en respuestas antes que en preguntas. Enseñamos desde el norte desarrollado (el norte metido en nuestra cabeza), en las condiciones del sur empobrecido.

En segundo lugar, la lógica del modelo de competencias corresponde a un conjunto de necesidades propias de la etapa neoliberal del desarrollo capitalista; entonces estamos llevando a cabo procesos de enseñanza-aprendizaje no sólo que miran al pasado, sino desde una realidad que no corresponde a la realidad latinoamericana y, de manera particular, a la mexicana. Esto significa que estamos enseñando saberes que ni siquiera han sido racionalizados en función de las condiciones propias en que viven nuestros estudiantes, sin ni siquiera haberlas cuestionado $y$, mucho menos, sin que hayamos formulado preguntas básicas sobre la pertinencia de esos saberes.

Los procesos de enseñanza-aprendizaje, en esas condiciones, se dan entre dos entidades inamovibles: quien enseña y quien aprende. Es 
decir, una relación sujeto-objeto mecánica que reproduce la relación de sojuzgamiento; el que sabe, el profesor y el que no sabe, el alumno. Esta es una de las características dominantes de nuestra práctica docente.

Alguien podría decir que es obligado seguir esos lineamientos porque están asociados a la asignación de presupuestos y eso es correcto, también se podría decir que lo que aprendimos son los paradigmas teóricos y metodológicos occidentales, pues no hay otros, y esto también, de algún modo, es razonable. Sin embargo, el problema es que lo asumimos ipor comodidad? De antemano como el único posible, lo que tiene consecuencias que afectan la función social de la educación. Un problema adicional es que renunciamos a ejercer la capacidad de reflexión y cuestionamiento de nuestra realidad y de nuestra propia práctica académica.

\section{$\mathbf{\xi} 3$}

Lo anterior nos conduce a reflexionar sobre nuestro quehacer como docentes, ya sea en licenciatura o en posgrado. En muchos casos nuestra práctica está dominada por la ausencia de cuestionamientos y la abundancia de certezas de nuestros saberes, lo cual tiene que ver con nuestras trayectorias académicas, con nuestras disposiciones teórico-metodológicas, como estructuras-estructurantes, en el sentido en el que Bourdieu (2003, p. 34) lo señala:

La definición de los problemas y la metodología de investigación utilizada procede de una tradición profesional de teorías, métodos y de competencias que solo pueden adquirirse al cabo de una formación prolongada. Las reglas del método científico tal como son explicitadas por los lógicos no corresponden con la realidad de las prácticas. Al igual que en otras profesiones, los científicos dan por supuesto que las teorías y métodos existentes son válidos y los utilizan para sus necesidades. No trabajan en el descubrimiento de nuevas teorías, sino en la solución de unos problemas concretos considerados como enigmas.

ITINERARIO EDUCATIVO • AÑO XXVI, N. ${ }^{\circ} 59 \cdot 3 I-5 I \cdot$ ENERO - JUNIO DE ZOID 
Así que, lo que regularmente enseñamos son esas disposiciones largamente aprendidas y ratificadas como válidas en la práctica docente o de investigación. Lo que esto significa es la reproducción de esquemas de poder, de puntos de observación y referencia que van encaminados a configurar estructuras-estructurantes de los saberes adquiridos y transmitidos. No quiere decir esto que dichos saberes no sean pertinentes, lo que quiere decir es que son saberes que enseñamos sin otros referentes y sin reflexión ni análisis de lo que representan en términos del origen de la información, conocimiento del contexto en el que se producen y en el que se hacen operativos.

Por su parte, son relaciones de poder porque se reproduce una relación de dominación entre el que sabe y enseña y el que no sabe y aprende; esto supone que la articulación de la relación sujetoobjeto se da de manera unidireccional. Por otra parte, también supone asumir los esquemas teóricos y los recursos metodológicos provenientes de los centros hegemónicos como los únicos válidos y pertinentes.

Este es un punto crítico, tanto en lo que se refiere a las prácticas docentes dominantes como a la imposición de programas institucionales expresados en políticas de gobierno. Dicho de otra manera, los programas institucionales para la educación convierten las prácticas académicas en estructuras-estructurantes sobre la base de un modelo educativo que no da lugar a la crítica ni a la reflexión y al cuestionamiento o a otras posibilidades para reformular contenidos y adecuarlos a las condiciones y contextos en los que se hallan las universidades y la propia relación sujeto-objeto en el proceso de enseñanza-aprendizaje.

En este sentido, pareciera que la cuestión de la crisis educativa no tiene salida y que estuviéramos irremediablemente condenados a aceptar las disposiciones programáticas de las autoridades educativas del país y a repetir esquemas teóricos, metodología y 
modelos educativos impuestos desde intereses supranacionales. Así, el problema es insoluble en el corto tiempo.

Sin embargo, ante la ausencia de opciones de futuro es preciso regresar a lo básico; desde mi punto de vista, lo básico es regresar a la estrategia individual para inducir, al principio pequeñas trasformaciones en nuestros puntos de observación, en nuestras prácticas, en la formulación de nuevas y aventuradas preguntas; esto puede constituir el inicio de un movimiento molecular y, citando de nueva cuenta a Bachelard,

\begin{abstract}
se puede entonces plantear, como un principio general del racionalismo experimental, la necesidad de reformar la experiencia primera: todas las formas supra racionales deben ser producidas por reformas intelectuales...todo descubrimiento real determina un método nuevo, por tanto debe arruinar el método anterior. Dicho de otro modo, en el reino del pensamiento la imprudencia es un método. Sólo la imprudencia puede tener éxito (1973, p. 11-12).
\end{abstract}

Lo que plantea Bachelard tiene que ver con la necesidad de romper con los esquemas impuestos, a través de la reflexión y el análisis riguroso tanto de nuestras propias prácticas y concepciones como con los contenidos programáticos y con ello recuperar todo aquello recuperable de acuerdo a la realidad en la cual operamos. De la renuncia a la crítica pasar a la transformación del pensamiento y con ello a la transformación de la práctica académica.

Dicho en otros términos, de lo que se trataría sería de pensar y definir estrategias didáctico-pedagógicas acordes a nuestra realidad y revisar críticamente los paradigmas teóricos y modelos dominantes. Esto significaría transformar la relación sujeto que enseña y objeto que aprende y establecer una relación dialéctica entre el sujeto y el objeto, en la cual el sujeto es al mismo tiempo objeto y viceversa. Lo básico, entonces, sería la construcción del sujeto, de los sujetos del proceso de aprendizaje.

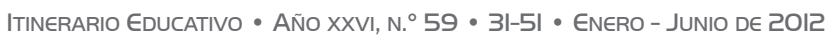


De esta forma, aquello que aparece como extremadamente difícil de cambiar se puede transformar desde el propio sujeto. Se invertiría, pues, el proceso: en lugar de esperar los cambios desde arriba, se empezaría a generar desde abajo, insistimos, en un movimiento molecular. Así la práctica docente (en licenciatura o en posgrado) se puede transformar desde la propia realidad del docente, en su condición de sujeto. Este es un proceso que tiene que ver con lo que Hugo Zemelman (2005) llama la voluntad de conocer.

La voluntad de conocer no es una cuestión declarativa, en el sentido del mero deseo de aprender, sino que constituye la voluntad de construirse como sujeto y con ello de asumir el compromiso de revisar de manera permanente cómo pensamos, qué pensamos y cómo desde ese pensamiento construimos objetos de conocimiento y de enseñanza. Desde esta lógica, se entiende que no hay objeto sin sujeto. Para que quien piensa se convierta en sujeto debe tener la disposición para transformar su pensamiento a partir de una serie de rupturas epistemológicas que lo acercan a la dialéctica de la relación sujeto-objeto. Este proceso complejo no es tan complicado pero sí puede resultar doloroso cuando nos percatamos de que nuestras certezas teóricas, metodológicas o pedagógicas se diluyen y no nos alcanzan para explicar una realidad que se encuentra permanentemente transcurriendo.

Sobre este compromiso con la voluntad de conocer, Bachelard (2002, p.105-107) plantea que

el sujeto entendido como factor de rectificación, como principio de enderezamiento por la voluntad y el pensamiento, pone necesariamente en duda la voluntad anterior, la conciencia anterior...El sujeto, al meditar sobre el objeto, elimina no solo los rasgos irregulares de este sino también actitudes irregulares de su propio comportamiento intelectual. El sujeto elimina sus singularidades, tiende a convertirse en objeto para sí mismo...un sujeto se aprende de manera clara y distinta solo porque ha podido rectificarse de punta a punta. 
Visto así, cada transformación de las irregularidades de nuestras propias concepciones y prácticas académicas supone una ruptura epistemológica y a su vez una reforma de nuestro pensamiento.

Esta misma aproximación se puede aplicar tanto para la investigación científica como para la práctica docente. En esta el maestro se objetiva, es decir, busca expresar de una manera pertinente un conjunto de saberes con la pretensión de que el alumno los entienda. Digamos que esa es la mecánica del proceso de enseñanzaaprendizaje. Las características de nuestra propia práctica ya están permeadas por saberes definidos y nosotros lo que hacemos es reproducirlos en el aula. Es decir, lo que reproducimos es un tipo de proceso que implica una relación sujeto-objeto que hace operativo el proceso de enseñanza-aprendizaje sobre el cual no reflexionamos. Este tipo de práctica docente nos conduce a reproducir todo lo bueno y fundamentado que aprendimos pero también todos los errores, certezas incuestionadas y deformaciones tanto de los saberes como de nuestro propio pensamiento.

Hay aquí un círculo vicioso en el cual impera la visión de lo que el maestro ha aprendido en su formación académica, o sea, un conjunto de saberes que se convierten en la visión dominante. Eso es lo que enseñamos y pocas veces cuestionamos esos saberes y nuestra propia práctica, porque el secreto está en no perder la certezas para seguir manteniendo la dominación y la legitimidad como buen maestro. Por ello, cuando cuestionamos no nos cuestionamos a nosotros mismos en cuanto a nuestra propia formación y nuestra propia práctica.

Entonces, parafraseando a Boaventura de Sousa, lo que aprendemos y lo que enseñamos es una forma de ver el mundo y junto con ello lo que se tiene que ver, una forma de ver incuestionada; pero resulta que lo que no vemos es infinitamente mayor y más rico que lo que aprendemos y enseñamos a ver. De esta manera, reproducimos las estructuras-estructurantes que nos legitiman

ITINERARIO EDUCATIVO • AÑO XXVI, N. ${ }^{\circ} 59 \cdot 3 I-5 I \cdot$ ENERO - JUNIO DE ZOIS 
frente a nuestros colegas, frente a los estudiantes o frente a los directivos. Llegar al punto de ver lo que no vemos requiere del cuestionamiento sobre nuestra forma de pensar, sobre nuestra trayectoria intelectual y con ello sobre nuestra forma de investigar o de enseñar.

Siguiendo con la paráfrasis, es incalculablemente más lo que podemos aprender que lo que podemos saber y, en el mismo sentido, es incalculablemente más lo que podemos aprender que lo que podemos enseñar. Lo anterior nos lleva a pensar el proceso de enseñanza-aprendizaje de una manera totalmente diferente no entre sujeto-objeto sino entre sujetos que construyen objetos de aprendizaje. Nos hace falta hacer del proceso educativo una relación dialéctica en la cual se confronten dos sujetos ante la necesidad de construir el objeto de aprendizaje: por un lado un sujeto que sabe y aprende mientras enseña y, por otro, un sujeto que aprende y a través del cuestionamiento enseña. Se trata de una construcción de sujetos en tanto no están dados.

Para lograr lo anterior se requiere, nuevamente utilizando la paráfrasis, como lo señalaría Zemelman, de la voluntad no sólo de conocer sino de la voluntad de aprender. Así, en el contexto de la crisis global del capitalismo y de las resistencias de los grupos oligárquicos mundiales y ante la incapacidad de todavía no poder estructurar opciones de futuro, se hace necesario regresar a lo básico, a la construcción de sujetos como alternativa para darle un nuevo impulso a la historia desde la educación, desde nuestra propia práctica docente y de investigación. Como dice Bachelard «sólo cerrando los ojos nos preparamos para ver» (2002, p 109). Este es el sentido de la epistemología crítica.

\section{Referencias}

Bachelard, Gastón. (1999). La formación del espíritu científico. México: Siglo XXI. 
Bachelard, Gastón. (1998). El compromiso racionalista. México: Siglo XXI.

Bachelard, Gastón. (2002). Estudios. Argentina: Amorrortu.

Bloch, Ernest. (2007). El principio esperanza (I). España: Trotta.

Bourdieu, Pierre. (2003). El oficio de científico, ciencia de la ciencia y reflexividad. España: Anagrama.

De Sousa Santos, B. (2009). Una epistemología del sur. Argentina: CLACSO-Siglo XXI.

Perrenoud, Philippe. (2004). «La clave de los campos sociales: competencias del actor autónomo, o cómo evitar ser abusado, aislado, dominado o explotado cuando no se es rico ni poderoso». En Simone Rychen, D., y Hersh Salganiik, L. Definir y seleccionar competencias fundamentales para la vida. México: FCE.

Zemelman, Hugo. (2005). Voluntad de conocer, el sujeto y su pesamiento en el paradigma crítico. España: Anthropos. 